\title{
DECLARACIÓN DE ALMA-ATA
}

$\mathrm{L}$ a declaración de Alma-Ata marca un hito en la salud pública mundial al introducir el concepto de salud -entendida como el estado de bienestar físico, mental y social- como un derecho fundamental y como una responsabilidad global compartida entre los gobiernos y la población. El documento presenta el concepto de atención primaria de la salud como la asistencia sanitaria esencial universal basada en métodos y tecnologías prácticas, científicamente fundadas y socialmente aceptables. Lamentablemente, la declaración ha pasado de ser un imperativo global a un ideal casi olvidado.

La meta de un nivel aceptable de salud para toda la humanidad en el año 2000 evidentemente no ha sido alcanzada y ello invita a una reflexión profunda sobre nuestro rol en lograr que este objetivo no sea visto como una utopía sino como una necesidad y un deber.

Consideramos que a casi 35 años de la publicación del documento original, es pertinente refrescar la memoria de todos los involucrados e instar a las instituciones de educación médica a incluir las ideas de la declaración como parte fundamental del proceso formativo en ciencias de la salud. Con la reproducción de la declaración a propósito del presente número de la Revista Peruana de Medicina Experimental y Salud Pública, dedicado a la atención primaria de la salud, esperamos contribuir a su rescate y difusión en bien de toda la población.

Alonso Soto Tarazona MD, MSc, PhD Miembro del Comité Editor RPMESP

\section{CONFERENCIA INTERNACIONAL SOBRE ATENCIÓN PRIMARIA DE SALUD, ALMA-ATA, URSS, 6-12 DE SEPTIEMBRE DE $1978^{*}$}

La Conferencia Internacional sobre Atención Primaria de Salud, reunida en Alma-Ata en el día de hoy, doce de septiembre de mil novecientos setenta y ocho, considerando la necesidad de una acción urgente por parte de todos los gobiernos, de todo el personal de salud y de desarrollo y de la comunidad mundial para proteger y promover la salud de todos los pueblos del mundo, hace la siguiente Declaración.

\section{I}

La Conferencia reitera firmemente que la salud, estado de completo bienestar físico, mental y social, y no solamente la ausencia de afecciones o enfermedades, es un derecho humano fundamental y que el logro del grado más alto posible de salud es un objetivo social sumamente importante en todo el mundo, cuya realización exige la intervención de muchos otros sectores sociales $y$ económicos, además del de la salud.
II

La grave desigualdad existente en el estado de salud da la población, especialmente entre los países en desarrollo y los desarrollados, así como dentro de cada país, es política, social y económicamente inaceptable $y$, por tanto, motivo de preocupación común para todos los países.

\section{III}

El desarrollo económico y social, basado en un Nuevo Orden Económico Internacional, es de importancia fundamental para lograr el grado máximo de salud para todos y para reducir el foso que separa, en el plano de la salud, a los países en desarrollo de los países desarrollados. La promoción y protección de la salud del pueblo es indispensable para un desarrollo económico y social sostenido y contribuye a mejorar la calidad de la vida y a alcanzar la paz mundial.
IV

El pueblo tiene el derecho y el deber de participar individual y colectivamente en la planificación y aplicación de su atención de salud.

\section{$\mathrm{V}$}

Los gobiernos tienen la obligación de cuidar la salud de sus pueblos, obligación que sólo puede cumplirse mediante la adopción de medidas sanitarias y sociales adecuadas. Uno de los principales objetivos sociales de los gobiernos, de las organizaciones internacionales $y$ de la comunidad mundial entera en el curso de los próximos decenios debe ser el de que todos los pueblos del mundo alcancen en el año 2000 un nivel de salud que les permita llevar una vida social y económicamente productiva. La atención primaria de salud es la clave para alcanzar esa meta como parte del desarrollo conforme al espíritu de la justicia social.

\footnotetext{
* Publicado originalmente por la Organización Mundial de la Salud,

Disponible en: http://www.who.int/publications/almaata_declaration_en.pdf
} 
contra las enfermedades endémicas locales; el tratamiento apropiado de las enfermedades y traumatismos comunes; y el suministro de medicamentos esenciales;

sanition primaria de salud es la asistencia sanitaria esencial basada en métodos tecnologías prácticos, científicamente fundados y socialmente aceptables, puesta al alcance de todos los individuos y familias de la comunidad mediante su plena participación y a un costo que la comunidad y el país puedan soportar, en todas y cada una de las etapas de su desarrollo con un espíritu de autorresponsabilidad y autodeterminación. La atención primaria forma parte integrante tanto del sistema nacional de salud, del que constituye la función central y el núcleo principal, como del desarrollo social y económico global de la comunidad. Representa el primer nivel de contacto de los individuos, la familia y la comunidad con el sistema nacional de salud, llevando lo más cerca posible la atención de salud al lugar donde residen y trabajan las personas, $y$ constituye el primer elemento de un proceso permanente de asistencia sanitaria.

\section{VII}

La atención primaria de salud:

1. Es a la vez un reflejo y una consecuencia de las condiciones económicas y de las características socioculturales y políticas del país $y$ de sus comunidades, y se basa en la aplicación de los resultados pertinentes de las investigaciones sociales, biomédicas y sobre servicios de salud y en la experiencia acumulada en materia de salud pública;

2. Se orienta hacia los principales problemas de salud de la comunidad y presta los servicios de promoción, prevención, tratamiento y rehabilitación necesarios para resolver esos problemas;

3. Comprende, cuando menos, las siguientes actividades: la educación sobre los principales problemas de salud y sobre los métodos de prevención y de lucha correspondientes; la promoción del suministro de alimentos y de una nutrición apropiada, un abastecimiento adecuado de agua potable y saneamiento básico; la asistencia materno infantil, con inclusión de la planificación de la familia; la inmunización contra las principales enfermedades infecciosas; la prevención y lucha
4. Entraña la participación, además del sector sanitario, de todos los sectores y campos de actividad conexos del desarrollo nacional y comunitario, en particular la agricultura, la zootecnia, la alimentación, la industria, la públicas, las comunicaciones y otros sectores y exige los esfuerzos coordinados de todos esos sectores;

5. Exige y fomenta en grado máximo la autorresponsabilidad y la participación de la comunidad y del individuo en la planificación, la organización, el funcionamiento y el control de la atención primaria de salud, sacando el mayor partido posible de los recursos locales y nacionales y de otros recursos disponibles, y con tal fin desarrolla mediante la educación apropiada la capacidad de las comunidades para participar:

6. Debe estar asistida por sistemas de envío de casos integrados, funcionales y que se apoyen mutuamente, a fin de llegar al mejoramiento progresivo de la atención sanitaria completa para todos, dando prioridad a los más necesitados;

7. Se basa, tanto en el plano local como en el de referencia y consulta de casos, en personal de salud, con inclusión según proceda, de médicos, enfermeras, parteras, auxiliares $y$ trabajadores de la comunidad, así como de personas que practican la medicina tradicional, en la medida que se necesiten, con el adiestramiento debido en lo social y en lo técnico, para trabajar como un equipo de salud y atender las necesidades de salud expresas de la comunidad.

\section{VIII}

Todos los gobiernos deben formular políticas, estrategias y planes de acción nacionales, con objeto de iniciar y mantener la atención primaria de salud como parte de un sistema nacional de salud completo y en coordinación con otros sectores. Para ello, será preciso ejercer la voluntad política para movilizar los recursos del país y utilizar racionalmente los recursos externos disponibles. educación, la vivienda, las obras
IX

Todos los países deben cooperar, con espíritu de solidaridad y de servicio, a fin de garantizar la atención primaria de salud para todo el pueblo, ya que el logro de la salud por el pueblo de un país interesa y beneficia directamente a todos los demás países. En este contexto, el informe conjunto OMS/UNICEF sobre atención primaria de salud constituye una base sólida para impulsar el desarrollo y la aplicación de la atención primaria de salud en todo el mundo.

\section{X}

Es posible alcanzar un nivel aceptable de salud para toda la humanidad en el año 2000 mediante una utilización mejor y más completa de los recursos mundiales, de los cuales una parte considerable se destina en la actualidad a armamento y conflictos militares. Una verdadera política de independencia, paz, distensión y desarme podría y debería liberar recursos adicionales que muy bien podrían emplearse para fines pacíficos y en particular para acelerar el desarrollo social y económico asignando una proporción adecuada a la atención primaria de salud en tanto que elemento esencial de dicho desarrollo.

La Conferencia Internacional sobre Atención Primaria de Salud exhorta a la urgente y eficaz acción nacional e internacional a fin de impulsar y poner en práctica la atención primaria de salud en el mundo entero y particularmente en los países en desarrollo, con un espíritu de cooperación técnica y conforme al Nuevo Orden Económico Internacional. La Conferencia insta a los gobiernos, a la OMS y al UNICEF y a otras organizaciones internacionales, así como a los organismos internacionales, así como a los organismos multilaterales y bilaterales, a las organizaciones no gubernamentales, a los organismos de financiación, a todo el personal de salud y al conjunto de la comunidad mundial, a que apoyen en el plano nacional e internacional el compromiso de promover la atención primaria de salud y de dedicarle mayor apoyo técnico y financiero, sobre todo en países en desarrollo. La Conferencia exhorta a todas las entidades antedichas a que colaboren en el establecimiento, el desarrollo y el mantenimiento de la atención primaria de salud de conformidad con el espíritu y la letra de la presente Declaración. 